\title{
La femme, le chien et le clerc
}

\author{
Article
}

Accepted Version

Le Saux, F. (2017) La femme, le chien et le clerc. Reinardus, 28 (1). pp. 130-141. ISSN 1569-9951 doi:

https://doi.org/10.1075/rein.28.09les Available at https://centaur.reading.ac.uk/68702/

It is advisable to refer to the publisher's version if you intend to cite from the work. See Guidance on citing.

Published version at: http://www.jbe-platform.com/content/journals/15699951

To link to this article DOI: http://dx.doi.org/10.1075/rein.28.09les

Publisher: John Benjamins Publishing

All outputs in CentAUR are protected by Intellectual Property Rights law, including copyright law. Copyright and IPR is retained by the creators or other copyright holders. Terms and conditions for use of this material are defined in the End User Agreement.

\section{www.reading.ac.uk/centaur}

\section{CentAUR}

Central Archive at the University of Reading

Reading's research outputs online 
La femme, le chien et le clerc: le Dame Sirith and the Weeping Bitch moyen-anglais

Françoise Le Saux

Résumé:

Cette étude explore le réseau symbolique entre femme et animal dans le fabliau moyen-anglais Dame Sirith and the Weeping Bitch, et offre une réinterprétation du personnage de Margery à la lumière du Liber vaccae. Cet ouvrage (pseudo-)scientifique bien connu dans les milieux universitaires dès le début du XIIIe siècle atteste une croyance en la possibilité de transformer un être humain en animal: la crédulité de la jeune femme relèverait ainsi d'un trait de société, tout autant que d'une faiblesse personnelle. Les changements apportés par Caxton à sa version du fabliau - en particulier, sa substitution d'une chienne par une chatte dans son récit de la ruse de l'entremetteuse, et un retour au context païen de l'histoire telle que la relate Petrus Alfonsi - sont révélateurs d'un désir de distanciation par rapport à une thématique plus dérangeante que ne l'aurait soupçonné le lecteur du XXIe siècle.

Dame Sirith and the Weeping Bitch est un des plus anciens fabliaux à nous être parvenus en moyen-anglais, transmis dans un manuscrit unique (Oxford, MS Digby 86) datant du dernier quart du XIIIe siècle. ${ }^{1}$ L'intrigue est tirée, plus ou moins directement, de l'exemplum XIII de la Disciplina clericalis de Petrus Alfonsi, illustrant 'mulierum ingenia', les ruses des femmes. ${ }^{2}$ Margery, une jeune femme bourgeoise, repousse les avances du clerc Wilekin, qui avait essayé de la séduire durant l'absence de son mari. Pour parvenir à ses fins, Wilekin a recours aux services de Dame Sirith, une vieille entremetteuse, laquelle convainc Margery de se donner au jeune homme grâce à un ingénieux stratagème: elle fait manger à sa petite chienne une pâtée poivrée qui lui fait couler les yeux, et fait croire à Margery qu'il s'agit là de sa fille mariée, transformée en chien par un clerc éconduit et pleurant à chaudes larmes sur son sort.

Le fabliau moyen-anglais est court - 450 vers - et avec 400 vers de dialogue, a des allures d'interlude théâtral. ${ }^{3}$ Dame Sirith s'adresse ainsi à sa chienne en discours direct alors qu'elle la fait manger (vers 279-284):

\footnotetext{
${ }^{1}$ Le manuscrit, daté vers 1275 , contient également une version en moyen anglais de l'histoire du renard dans le puits. Le fabliau est un genre sous-représenté dans la littérature en moyen-anglais; cf. Keith Busby, "Dame Sirith and De Clerico et Puella," in Companion to Early Middle English Literature, ed. N. H. G. E. Weldhoen et H. Aertsen (Amsterdam: Free Press, 1995), 67-78. Sur le manuscrit, voir Judith Tschann et Malcolm B. Parkes, Facsimile of Oxford Bodleian Library, MS Digby 86, Oxford, Oxford University Press, 1996.

2 "De canicula lacrimante," in Die Disciplina Clericalis des Petrus Alfonsi (das älteste Novellenbuch des Mittelalters), ed. Alfons Hilka et Werner Söderhjelm (Heidelberg: Carl Winter, 1911), 18-19. Sur l'importance de Petrus Alfonsi pour la dissémination de ce type d'histoires, voir John Tolan, Petrus Alfonsi and his Medieval Readers (Gainesville: University Press of Florida, 1993), 154-158.

${ }^{3}$ R. H. Robbins, "The English fabliau: before and after Chaucer," Moderna Sprák (1972): 235 (231-244). Voir aussi Michael Axton, European Drama of the early Middle Ages (Pittsburgh: University of Pittsburgh Press, 1975), 1724.
} 
"Pepir nou shalt thou eten

This mustart shal ben thi mete

And gar thin eien to rene;

I shal make a lesing

Of thin heie renning,

Ich wot wel wer and wenne."

"Maintenant il te faut manger du poivre; cette moutarde sera ta nourriture et te fera couler les yeux. Je concocterai un mensonge à propos de tes yeux qui coulent, je sais exactement où et quand." 4

Ce style dialogué donne en outre de la petite chienne l'image d'une interlocutrice silencieuse mais active de sa maîtresse dont elle obéit les ordres apparemment sans rechigner. Ceci contraste avecle texte de Petrus Alfonsi, où l'animal doit être affamé pendant deux jours pour lui faire avaler cette mixture:

Et caniculam quam apud se habebat duobus diebus ieiunare coegit et die tercio panem sinapi confectum ieiunanti largita est. Que dum gustaret, pre amaritudine oculi eius lacrimari coepit.

Et elle força une petite chienne qu'elle avait chez elle à jeûner pendant deux jours, et le troisième jour elle donna à l'animal qui n'avait rien mangé du pain pétri de moutarde. Quand la chienne en eut goûté, ses yeux se mirent à pleurer à cause de l'amertume du pain.

Dans les deux cas il s'agit du point de vue du lecteur du XXIe siècle d'un acte de cruauté, mais on notera l'absence de coercition dans le texte anglais, où il suffit à Dame Sirith de d'exprimer sa volonté pour être obéie. En termes de mise en scène, la petite chienne devient dans le fabliau anglais un personnage-clé, silencieusement présent aux côtés de Dame Sirith de manière continue du vers 166 à la fin du récit, et invitation constante à considérer les deux protagonistes féminins à la lumière d'une symbolique animale. C'est cet aspect que nous nous proposons d'explorer ici.

Regardons de plus près la canicula du récit. Nous y reconnaissons aisément un chien de petite race, de ceux que Brunetto Latini, dans le chapitre de Li Livres dou Tresor consacré aux chiens, nous décrit de la manière suivante: "Il a petis chiens gouços ki sont bons a garder maisons, et si i a trés petis camusos pour garder cambres et les lis as dames." Latini donne ensuite des informations quant aux techniques utilisées par les éleveurs pour que ces animaux soient "si petis et si briés ke merveille". Il s'agit donc de l'animal de compagnie de l'entremetteuse, familière de la chambre à coucher de sa maîtresse ${ }^{6}{ }^{6}$ un chien de dame, en

\footnotetext{
${ }^{4}$ Sauf indication contraire, les citations de 'Dame Sirith and the Weeping Bitch' sont tirées de The Trials and Joys of Marriage, ed. Eve Salisbury (Kalamazoo, Michigan: Medieval Institute Publications, 2002; accessible électroniquement: http://www.lib.rochester.edu/teams/text/salisbury-trrials-and-joys-dame-sirith). Traductions par mes soins.

${ }^{5}$ Brunetto Latini, Tresor, ed. Pietro G. Beltrami, Paolo Squillacioti, Plinio Torri et Sergio Vatteroni (Torino: Giulio Einaudi, 2007), Livre I, chapitre CLXXXIIII.

${ }^{6}$ Sur les animaux domestiques dans la sphère intime au moyen-âge, voir Kathleen Walker-Meikle, Medieval Pets (Woodbridge: Boydell Press, 2014), en particulier ch. 4, 'Living with Pets', 55-74.
} 
somme, et de surcroît femelle, ainsi que l'indique le suffixe féminin du latin canicula et que l'exige la suite du récit.

La petite chienne remplit des fonctions multiples qui dépassent de beaucoup son rôle important mais limité dans la diégèse. Simple animal, elle ne jouit pas moins d'une relation privilégiée avec Dame Sirith; elle obéit aveuglément à sa maîtresse, semble-t-il, et l'accompagne partout. Sa confiance est telle qu'elle accepte sans sourciller de manger la nourriture infâme que lui présente l'entremetteuse. La chienne de Dame Sirith préfigure ainsi le sort de Margery, qui finira par accepter un adultère auquel elle n'était guère encline. En même temps, l'animal est comme un prolongement de Sirith elle-même, une sorte de double canin à qui elle explique ses plans et qui semble comprendre ce qui doit être fait afin de gagner la "ful riche mede", la très riche récompense promise par le clerc éconduit. L'ambivalence du chien dans l'imaginaire médiéval - modèle de fidélité d'une part, animal impur de l'autre - permet de jouer sur les deux tableaux, invitant à une lecture des personnages en tension permanente.

Il convient de noter à ce propos que les personnages du fabliau anglais ne correspondent pas d'aussi près qu'on pourrait l'imaginer à ceux de l'exemplum de Petrus Alfonsi. Le mari dans le texte latin est un noble (“quidam nobilis progenie"), qui s'absente pour aller prier à Rome: dans le texte anglais, c'est un marchand qui se rend à la foire de Boston (Botolfston) dans le Lincolnshire. Le jeune homme, chez Petrus Alfonsi, est simplement un "iuиenis"; le fabliau anglais est plus précis (vers 3-6): il est "Ful modi mon and proud;/Wis he wes of lore / and gouthlich under gore / and clothed in fair sroud." ("Très fier et orgueilleux; il était instruit, bien fait de sa personne sous ses habits et bien vêtu").

Cette description a parfois été interprétée comme représentant Wilekin sous un jour noble et courtois $;{ }^{7}$ en fait on notera que l'accent mis sur son savoir indique d'emblée qu'il s'agit bien ici d'un clerc. Un clerc aisé, venant peut-être d'une bonne famille - d'où sa prestance et la qualité de son habillement - mais indéniablement un clerc: détail de poids dans une histoire de transformation animale aux relents de magie noire. De plus, ce clerc est certes contrarié par son manque de succès, mais sa tristesse ("drerimod he wente awai", "il partit tout triste") n'a aucune commune mesure avec celle du jeune homme du texte latin, dont la souffrance est telle qu'il tombe gravement malade ("nimio infirmitatis grauaretur"). ${ }^{8}$

L'entremetteuse anonyme de la Disciplina clericalis subit un changement plus discret mais crucial. Elle n'est plus décrite comme une vieille portant des habits de religieuse ("anus religionis habitu decorate"), et qui a accès à la jeune femme grâce à son apparence respectable ("pro magna religionis specie”, "à cause de son apparence de grande piété"). Dame Sirith se présente au contraire chez Margery comme une pauvre mendiante affamée et appelant la mort de ses vœux (vers 303-314). La vieille maquerelle retourne ainsi contre sa victime un louable acte de charité chrétienne à l'égard d'une personne dans le besoin. L'aspect particulièrement immoral, voire sacrilège, de ce stratagème appelle du reste les foudres du narrateur qui maudit Sirith de manière explicite (vers 331): "Crist awarie hire lif!", "Que Dieu la damne!"

Ce détail révèle un important changement dans le contexte culturel de l'histoire. Bien que Petrus Alfonsi ne le dise pas de manière explicite, les protagonistes du récit latin semblent bien être des païens. Le voyage du mari à Rome n'est pas décrit comme un pèlerinage, comme on s'y

\footnotetext{
${ }^{7}$ John Hines, The Fabliau in English (London and New York: Longman, 1993), 47-49.

${ }^{8}$ Les arguments avancés entre autres par R. E. Lewis, “The English fabliau tradition and Chaucer's Miller's Tale," Modern Philology 79 (1982): 241-255, sont réfutés par Thomas Honegger, "Curteisi' in Dame Sirith," Reinardus 15 (2002): 71-86, qui souligne l'effet comique de l'incongruité du lexique courtois.
} 
attendrait; il s'y rend simplement afin de prier, "oracionis studio", terme vague à dessein. Cette piété pas nécessairement chrétienne se retrouve chez sa belle et vertueuse épouse, qui accepte la visite d'une personne qu'elle ne connaît pas uniquement à cause de son habillement religieux. Détail révélateur, cette "religieuse" est mère d'une fille, sans que la jeune femme s'en étonne le moins du monde; et la vieille femme décrit la chasteté comme un crime ("culpa"), puni par la justice divine parce qu'ayant causé la maladie de l'amant éconduit. 'De canicula lacrimante' devient ainsi une variation plaisante sur le thème des métamorphoses des dieux du panthéon préchrétien, expliquant dans une certaine mesure la crédulité de la jeune femme. C'est parce qu'elle a le respect de sa religion païenne que la ruse peut réussir.

Dame Sirith, par contre, opère dans un cadre résolument chrétien: Margery émaille sa conversation de références explicites à Dieu et au Rédempteur. Le passage où elle offre à la vieille mendiante de quoi boire et manger est particulièrement riche de ce point de vue (vers 315330):

"Seli wif, God thee hounbinde!

Todai wille I thee mete finde

For love of God.

I have reuthe of thi wo

[...]

Com herin, ich wile thee fede,

God Almightten do thee mede,

And the Louerd that wes on Rode i-don,

And faste fourti daiis to non

And hevene and erthe haveth to welde.

As thilke Louerd thee foryelde,

Have her fles and eke bred,

And make thee glad, hit is mi red,

And have her the coppe with the drinke;

God do thee mede for thi swinke."

"Bonne femme, que Dieu te vienne en aide! Aujourd'hui je te trouverai à manger, pour l'amour de Dieu. J'ai pitié de ta souffrance. [...] Entre, je te donnerai à manger; que Dieu Tout Puissant te soit généreux, ainsi que Notre Seigneur qui fut mis en croix et jeûna quarante jours jusqu'à la neuvième heure, et règne sur le ciel et la terre. Au nom de ce même Seigneur qui t'a rachetée, mange cette viande et aussi ce pain, et mets-toi à l'aise, je t'en prie, et bois à cette coupe; que Dieu te recompense pour tes efforts."

Nous sommes loin ici d'une visite mondaine. Margery est en train d'offrir l'aumône à une pauvre vieille. Son geste relève explicitement du devoir d'assistance aux démunis (vers 316-317) et se place dans une optique résolument christique. Dame Sirith n'est pas accueillie parce qu'elle est respectable, mais à cause de sa souffrance présumée qui fait d'elle une figura du Christ agonisant. Dans les deux textes, l'entremetteuse exploite les croyances religieuses de la jeune femme pour lui parler; mais alors que dans l'exemplum latin, c'est justement le contexte de paganisme qui lui permet de convaincre sa victime, dans le fabliau moyen-anglais la piété de Margery constituerait plutôt un obstacle supplémentaire à surmonter. Même la plus écervelée des personnages féminins médiévaux aurait eu peine à croire que Dieu pouvait punir la chasteté par 
la métamorphose animale: l'argument de la vieille devait donc être modifié. À la menace de la justice divine se substitue ainsi la crainte de la nécromancie.

Le fait que Wilekin est un clerc prend à ce moment tout son sens, dans la mesure où, contrairement à ce que certains ont pu penser, ${ }^{9}$ ce détail donne un élément de vraisemblance au risque mentionné par Dame Sirith. En témoigne le scabreux Liber vaccae, traduit de l'arabe au début du XIIIe siècle et l'objet des foudres de l'évêque de Paris Guillaume d'Auvergne (1190?1249) dans son De Universo creaturarum (1231), preuve de la popularité de ce livre dans les milieux universitaires parisiens. ${ }^{10}$ Nombre de recettes contenues dans ce traité 'scientifique' ont pour but de donner à des êtres humains une forme animale. Une procédure complexe nécessitant des animaux femelles, diverses substances minérales et organiques, ainsi que le sperme de l'expérimentateur lui-même, était censée résulter en la naissance d'une créature dont les organes avaient, entre autres propriétés, des vertus transformatrices. ${ }^{11}$ De plus, ce traité donne à son utilisateur des instructions sur la manière de se présenter, afin de mieux impressionner la clientèle: un élément de poudre aux yeux à des fins ouvertement commerciales. ${ }^{12}$ En cette fin du XIIIe siècle, Margery n'aurait pas été la seule à croire en la possibilité d'être transformée en animal. ${ }^{13}$

Les craintes suscitées par Dame Sirith sont d'autant plus compréhensibles qu'elles sont explicitement liées à une peur de la sorcellerie. La nécromancie est suggérée dans la Disciplina clericalis déjà, mais il s'agit d'un usage métaphorique. Le disciple, ayant entendu l'histoire, s'exclame: "Hoc puto fieri arte diabolica", "Je pense que cela a été effectué par l'œuvre du diable", à quoi le maître répond, "Ne dubites!", 'N'en doute pas'. Toutefois, l'exemplum luimême ne nous montre qu'une vieille femme; rusée, certes, immorale et hypocrite, assurément, mais pas une sorcière. Dans le fabliau anglais, il en va autrement.

La réaction de Dame Sirith quand Wilekin vient la voir pour la première fois révèle que celle-ci a une réputation, et qu'il lui faut être prudente (vers 193-216):

'Benedicite! Be herinne

Her havest thou, sone, mikel senne.

Louerd, for His swete nome,

Lete thee therfore haven no shome!

\footnotetext{
${ }^{9}$ On mentionnera tout particulièrement John Hines, The Fabliau in English, 50, qui voit en Margery une simple écervelée. Thomas Honegger , "Curteisi in Dame Sirith," 78-82, discerne davantage de profondeur dans le personnage.

${ }^{10}$ Guilielmi Alverni Episcopi parisiensis, mathematici perfectissimi eximii philosophi ac theologi praestantissimi, Omnia opera (Ambiani, apud Michaelem du-Neuf-Germain, 1674); vol. 1, 593-1074. Il nous reste du Liber vaccae (aussi connu sous le nom de liber aneguemis ou liber Platonis) environ 14 manuscrits datant du XIIIe au XVIe siècle; le texte n'a pas encore fait l'objet d'une édition critique. Voir Dag Nikolaus Hasse, "Plato arabico-latinus: Philosophy - Wisdom Literature - Occult Sciences," in The Platonic Tradition in the Middle Ages. A Doxographic Approach, ed. Stephen Gersch et Maarten J. F. M. Hoenen (Berlin et New York: de Gruyter, 2002), 31-65.

${ }^{11}$ Voir Maaike van der Lugt, "“Abominable Mixtures': The Liber vaccae in the medieval West, or the dangers and attractions of natural magic," Traditio 64 (2009): 229-277. David E. Pingree, "Plato's Hermetic Book of the Cow", in Il Neoplatonismo nel Rinascimento, ed. Pietro Prini (Roma: Istituto della Enciclopedia Italiana, 1993), 133-145. ${ }^{12}$ Van der Lugt, “Abominable Mixtures," 239, relève l'aspect théâtral de certaines des 'recettes' du Liber vaccae.

${ }^{13}$ L'exemple le plus répandu d'une telle croyance est celui du loup-garou, que Guillaume d'Auvergne, dans son $D e$ Universo II, 3, 13 prend suffisamment au sérieux pour démontrer que la transformation n'était pas physique; voir Gaël Milin, Les Chiens de Dieu, La representation du loup-garou en Occident (XIe-XXe siècles), Brest: Centre de recherche bretonne et celtique, 1993 (Cahiers de Bretagne Occidentale 13), 66-67. La croyance en une transformation, bien physique, en chien ou en chat est également attestée; voir Frank Hamel, Human Animals (New York: Frederick A. Stokes, 1915).
} 
Thou servest affter Godes grome, Wen thou seist on me sil blame.

For ich am old, and sek and lame;

Seknesse haveth maked me ful tame.

Blesse thee, blesse thee, leve knave,

Leste thou mesaventer have,

For this lesing that is founden

Oppon me that am harde i-bonden.

Ich am on holy wimon,

On wicchecrafft nout I ne con,

Bote with gode men almesdede

Ilke dai mi lif I fede,

And bidde mi Pater Noster and mi Crede,

That Goed hem helpe at hore nede,

That helpen me mi lif to lede.

His lif and his soule worthe $\mathrm{i}$-shend

That thee to me this hernde haveth send;

And leve me to ben i-wreken

On him this shome me haveth speken.

(Benedicite! Tu as commis ici un grand péché, mon fils. Que le Seigneur, par son doux nom, t'accorde de ne pas avoir à en souffrir! Tu mérites le courroux de Dieu, de dire sur mon compte des choses si vilaines, car je suis vieille et malade et impotente; la maladie m'a bien diminuée. Signe-toi, signe-toi, cher garçon, qu'il ne t'arrive pas de mésaventure à cause de ce mensonge à mon encontre, moi qui suis dans la pénurie. Je suis une sainte femme, je n'y connais rien en sorcellerie, mais je vis chaque jour des aumônes d'hommes bons, et je prie mon Notre Père et mon Crédo qu'au moment de leur besoin, Dieu aide ceux qui m'aident à survivre, et qu'Il leur accorde Sa protection. Qu'il soit honni, corps et âme, celui qui t'a envoyé à moi pour cette affaire, et que je sois vengée de celui qui a dit de moi cette calomnie.

Cette réaction semble excessive. Wilekin a certes laissé entendre qu'il songeait au suicide, mais ce qui inquiète Sirith n'est pas la possibilité que le jeune homme meure en état de péché mortel; elle se préoccupe bien davantage d'être accusée de sorcellerie. Or, Wilekin n'a fait appel qu'à "thine crafftes and thine dedes" (vers 191), termes vagues qu'on pourrait traduire simplement par "tes services". L'explosion de la vieille, assortie de malédictions fort peu en accord avec l'image de sainte femme qu'elle essaie de projeter, tendrait à suggérer qu'elle anticipe le besoin de se protéger. Effectivement, une fois rassurée sur la bonne foi et la discrétion de son nouveau client, Sirith révèle de manière explicite sa crainte du tribunal ecclésiastique:

For al the world ne wold I nout

That ich were to chapitre i-brout

For none selke werkes.

Mi jugement were sone i-given

To ben with shome somer driven

With prestes and with clarkes. (243-8) 
Pour rien au monde je ne voudrais comparaître devant le chapitre pour de telles actions. Je serais immédiatement condamnée à être promenée sur une mule, dans la honte, par les prêtres et les clercs.

Sirith n'a donc pas la conscience tranquille, et elle craint une révélation qui lui vaudrait la perte du revenu venant des aumônes. On notera l'habileté de l'entremetteuse dans son entretien avec Wilekin. Elle laisse entendre, en le niant un peu trop fort, qu'elle aurait peut-être une certaine expérience de la sorcellerie, et dit de manière explicite qu'elle craint le tribunal ecclésiastique, donnant ainsi à ses services des allures de mystère et d'interdit justifiant ses émoluments. ${ }^{14}$ Le risque pour la vieille entremetteuse est sans doute réel, mais nous avons surtout affaire à une mise en scène destinée à appâter et contrôler le client.

Le thème de la sorcellerie dans le fabliau est bien sûr focalisé sur la petite chienne de Sirith, sa supposée fille transformée en animal; la vieille affirme ainsi que l'amant éconduit de sa fille avait "bigon to wiche", "eu recours à la nigromancie". Le succès de la ruse et son potentiel comique dépendent en grande partie de la vraisemblance de la situation. La fidélité du chien à son maître est la caractéristique première de l'animal dans les bestiaires, ainsi que dans des récits tels que 'canis' dans la tradition des Sept Sages de Rome, ${ }^{15}$ il n'y a donc rien d'étonnant à ce que la petite chienne de Sirith suive docilement sa maîtresse malgré l'inconfort dû à sa pâtée épicée. De même, il n'est pas rare qu'une dame parle à son animal de compagnie comme s'il était capable de la comprendre; les impératifs stylistiques de ce fabliau-interlude s'accommodent donc parfaitement avec l'observation quotidienne.

Au chien fidèle s'ajoute toutefois aussi l'image du chien impur, qui mange ce qu'il a vomi et lèche le pus des blessures d'un Lazare mendiant devant les portes des nantis. La canicula semble être une petite bête choyée n'ayant guère en commun avec les chiens errants aux habitudes peu ragoûtantes, mais ces connotations négatives se profilent en relation avec les deux personnages féminins. Prenons Margery. Fidèle à son mari en début de récit - qualité canine positive - elle va en fin de compte accueillir chaleureusement le prétendant qu'elle avait repoussé - parallèle potentiel avec le chien retournant à ses vomissures. En fin de récit, la jeune femme abandonne ses principes, sa dégradation morale figurant comme une littéralisation de l'équation faite par Sirith entre jeune femme et petite chienne. Quant à l'entremetteuse, elle se situe clairement dans un monde troublé, aux marges de la société; elle vit des vices des autres, léchant métaphoriquement les blessures de ses clients. Ceci dit, comme était censée le faire la langue du chien, elle les guérit: Wilekin est entièrement remis de sa mélancolie. Cette guérison est moralement douteuse, mais le jeune homme aura du moins échangé le risque d'un péché mortel (le suicide) avec un péché charnel, et donc véniel.

Le choix d'une chienne comme instrument de la déception d'une jeune épouse vertueuse était donc particulièrement heureux, rendant cette petite histoire éminemment adaptable à différentes audiences et susceptible d'interprétations diverses. C'est pourquoi la version que nous en donne l'imprimeur et traducteur anglais Caxton dans The Book callid Caton (1483) ne manque pas d'étonner, puisque la chienne y devient un chat. ${ }^{16}$

\footnotetext{
${ }^{14}$ Sur la nature de la punition crainte par Sirith, voir Middle English Literature, ed. Thomas J. Garbáty (Lexington, MA: D. C. Heath, 1984), 247 (442-454).

15 "C'est li examples dou levrier [canis]", Li Ystoire de la male marastre. Version M of the Roman des sept sages de Rome, ed. Hans R. Runte (Tübingen: Max Niemeyer Verlag, 1974), 14-17.

${ }^{16}$ L'ouvrage n'a pas encore fait l'objet d'une edition critique. La version Caxton de Dame Sirith citée ici est tirée de "Meretricem Fuge: Flee the Harlot," in The Oxford Book of Late Medieval Verse and Prose, ed. Douglas Gray (Oxford, NewYork: Oxford University Press, 1985), 15.C (229-231; notes 465).
} 
Contrairement au fabliau moyen-anglais, Caxton replace l'histoire dans un contexte d'érudition, citant d'emblée Petrus Alfonsi comme sa source: "Peter Alphons reherceth in his book...", "Petrus Alfonsi dit dans son livre..." L'anecdote est maintenant située en Espagne; le mari est un bourgeois, mais nulle mention n'est faite à un quelconque voyage; quant au clerc, il est présenté comme un soupirant de longue date. L'entremetteuse est une voisine, vivant "nyghe his hows", "près de chez lui" (ligne7), et c'est elle qui va le voir, parce qu'elle s'inquiète de le voir si mal en point. Il se fait qu'elle connaît aussi fort bien la bourgeoise. Marché conclu avec le clerc, le récit continue de la manière suivante (lignes 14-19):

This olde bawde had a lytel catte whiche she named Pasquette, the which she kepte wythout ony mete or drynke the space of thre dayes, and after she gave to the catte a lytel flesshe with ryght stronge mustard. And after, she wente forto speke wyth the sayd bourgeys wyf, and ledde with hir her lytel catte, but bycause that she had eten the sayd mustard she dyd none other but wepte ever.

La scène est pour le moins intrigante. Pour qui a la moindre expérience de ces animaux, il est peu convaincant qu'un chat souffrant de la sorte se laisse docilement mener d'un endroit à l'autre. Il est également frappant que dans une histoire où les protagonistes humains restent tous anonymes, ce chat (ou plutôt cette chatte) a un nom, Pâquette, lui donnant une identité propre. La mise en scène agencée par l'entremetteuse pour accéder à la jeune femme disparaît complètement, gommant les inégalités sociales entre les personnages, et remplacée par un dialogue (du reste fort réussi) où la bourgeoise pousse la vieille femme à lui dire pourquoi ellemême et sa chatte sont en pleurs.

L'explication donnée à la métamorphose de la fille en chat se rapproche de celle que nous trouvons chez Alfonsi: il s'agit d'une punition divine, "by the wylle and plesure of God". La jeune femme s'obstinait à se refuser au jeune homme qui l'aimait, "wherfore the goddes were wroth and turned hir into a catte, as ye may see." Le pluriel, "goddes", indique clairement que l'histoire est placée dans un contexte sinon pré-chrétien, du moins sarrasin, en accord avec la délocalisation du récit en une lointaine et exotique Espagne. Le côté choquant du subterfuge est atténué par cette distanciation, laquelle est soulignée par la répétition du mot "goddes" dans la suite du dialogue. La dame exprime sa crainte de partager le sort de la fille présumée de son interlocutrice "yf the goddes ben wrothe with me", ce à quoi la vieille renchérit qu'elle devrait se dépêcher de céder au clerc, "or the goddes be wrothe upon you." La jeune femme obtempère ainsi par crainte de la colère des dieux ("furour and wrath of the goddes", ligne 49), comme dans la Disciplina clericalis; mais la maquerelle de Caxton donne de surcroît à cette décision une justification d'ordre lignager: "For yf ye were tornyd into a lityl catte, ye shold be dyshonoured, and ye sholde be cause of the shame and dyshonoure perpeuell of al your lynage."

La crainte du déshonneur personnel et familial s'avère être un argument puissant, et fait que la bourgeoise accepte en son cœur ("consented wythin her herte", ligne 51) de céder au clerc. Comme, contrairement à l'exemplum latin, la jeune femme n'est pas dépeinte comme étant particulièrement pieuse, cette motivation supplémentaire donne un peu plus de crédibilité à sa décision. De surcroît, nous dit-on, cette personne est très crédule - elle "belevyd lightly" (ligne 35). Ce jugement est dans la droite ligne de la Disciplina clericalis, où la croyance en la magie est présentée comme une sottise; ${ }^{17}$ mais cet effort de rationalisation suggère également que Caxton éprouvait le besoin de donner un peu plus de vraisemblance à son histoire. Or, ce besoin

${ }^{17}$ On songera en particulier à l'exemplum xxiv, "De latrone et radio lunae” (ed. Hilka et Södershjelm, 35-36). 
d'atténuer l'aspect extravagant de la substitution de la chienne par une chatte n'est pas la moindre des faiblesses de la version Caxton. Une explication possible serait un modèle manuscrit corrompu; mais il semble que tel n'était pas le cas. Dougas Gray note que Caxton relate cette histoire dans un autre ouvrage, dans son recueil de fables d'Esope; ${ }^{18}$ là également, malgré le fait que sa source française mentionne de manière irréfutable qu'il s'agissait d'une chienne, l'animal de compagnie de l'entremetteuse est une chatte.

De toute évidence, Caxton estimait la version féline de l'histoire supérieure à la version canine. Peut-être le chien avait-il une image particulièrement valorisante parmi le lectorat visé par Caxton; cette préférence pourrait également être due à la détérioration de l'image du chat. Nous savons qu'à cette période, le chat est étroitement associé au culte satanique. Textes et manuscrits relatent des scenes de sabbat où les sorciers rendent hommage au diable sous forme de chat: une scène dépeinte dans certains manuscrits, tel celui de Grenoble, Bibliothèque Municipale, MS 352 rés (f.346 v.), où des sorcières adorent un gros chat tigré ${ }^{19}$ Se peut-il que Caxton ait trouvé le chat plus approprié que le chien à une histoire immorale dans un monde païen dominé par des dieux démoniaques? ${ }^{20}$ Toujours est-il que l'entremetteuse prend des allures de sorcière, et ce chat au comportement si peu naturel ressemble fort à son familier, alors même que Caxton se moque de la jeune femme qui croit en la magie. "Hoc puto fieri arte diabolica", s'exclamait le disciple dans le Disciplina Clericalis à propos de la ruse de la vieille. Presque trois siècles plus tard, Caxton semble avoir porté sur le personnage un regard fort similaire.

\footnotetext{
${ }^{18}$ Il s'agit des Fables of Alfonce, XI, où Caxton traduit le mot "chienne" de sa source par l'anglais "catte". Voir Gray, "Fuge meretricem", 465.

${ }^{19}$ Ce manuscrit, copié entre 1470 et 1482, est sensiblement contemporain du texte de Caxton. Le lien entre chat et forces diaboliques apparaît bien sûr beaucoup plus tôt, avec notamment la fameuse bulle papale Vox in Rama (13 juin 1233), où le pape Grégoire IX décrit une scène de sabbat comparable à celle dépeinte dans Grenoble, Bibliothèque Municipale, MS 352 rés. ; Epistolae saeculi XIII e regestis Pontificum Romanorum, ed. C. Rodenberg (Berlin: Weidmann, 1883; Monumenta Germaniae historica), 423-5 et 537.

${ }^{20}$ Les connotations diaboliques du chat sont bien attestées. Voir, entre autres, Nicholas J. Saunders, The Cult of the Cat (London: Thames and Hudson, 1991), et surtout Irina Metzler, "Heretical cats: Animal Symbolism in Religious Discourse", Medium Aevum Quotidianum 59 (2009): 16-32.
} 